\title{
The Impact of Palmaris Longus Muscle on Function in Sports: An Explorative Study in Elite Tennis Players and Recreational Athletes
}

\author{
Julie Vercruyssen ${ }^{1, *}$, Aldo Scafoglieri ${ }^{2}$ and Erik Cattrysse ${ }^{2}$ \\ 1 Faculty of Physical Education and Physiotherapy, Master of Science in Manual therapy, \\ Vrije Universiteit Brussel, Laarbeeklaan 103, 1090 Brussels, Belgium \\ 2 Faculty of Physical Education and Physiotherapy, Department of Experimental Anatomy, \\ Vrije Universiteit Brussel, Laarbeeklaan 103, 1090 Brussels, Belgium; \\ aldo.scafoglieri@vub.ac.be (A.S.); erik.cattrysse@vub.ac.be (E.C.) \\ * Correspondence: julievercruyssen@hotmail.com; Tel.: +32-472-741-808
}

Academic Editor: Giuseppe Musumeci

Received: 21 February 2016; Accepted: 24 March 2016; Published: 13 April 2016

\begin{abstract}
The Palmaris longus muscle can be absent unilateral or bilateral in about $22.4 \%$ of human beings. The aim of this study is to investigate whether the presence of the Palmaris longus muscle is associated with an advantage to handgrip in elite tennis players compared to recreational athletes. Sixty people participated in this study, thirty elite tennis players and thirty recreational athletes. The presence of the Palmaris longus muscle was first assessed using different tests. Grip strength and fatigue resistance were measured by an electronic hand dynamometer. Proprioception was registered by the Flock of Birds electromagnetic tracking system. Three tests were set up for measuring proprioception: joint position sense, kinesthesia, and joint motion sense. Several hand movements were conducted with the aim to correctly reposition the joint angle. Results demonstrate a higher presence of the Palmaris longus muscle in elite tennis players, but this was not significant. Maximal grip strength was correlated with gender and only on the dominant side; it was also correlated with age. Fatigue resistance showed only on the non-dominant side; a significant difference in elite and recreational athletes. In proprioception there was no correlation with elite and recreational athletes or the presence of the Palmaris longus muscle.
\end{abstract}

Keywords: grip strength; fatigue resistance; joint position sense; kinesthesia; joint motion sense; proprioception

\section{Introduction}

The Palmaris longus muscle (PLM) is described as probably the most variable muscle in the human body. Not only in terms of absence but also in variability of origin and insertion and morphology [1]. The PLM can be absent unilateral and bilateral in about $22.4 \%$ of human beings (Caucasians) with a range of $3.0 \%-63.9 \%$, depending on the ethnic background of a population [2]. A great diversity is reported between different populations in the prevalence of absence of the PLM [3].

Stecco et al. considers the PLM as a slender fusiform muscle with a short proximal belly and a long distal tendon (Figure 1). It is located in the most superficial layer of the anterior compartment of the forearm, between the radial and the ulnar flexors of the wrist [4]. In most cases, the distal tendon of the PLM shows a lateral slip that attaches to the superficial surface of the abductor pollicis brevis muscle that may contribute to thumb abduction [5]. In the proximal part of the forearm, the PLM is situated deep to the antebrachial fascia, while in the lower third of the forearm its tendon perforated the antebrachial fascia [4]. The origin of the PLM is at the epicondylus medialis of the humerus and the 
insertion is on the aponeurosis Palmaris [6]. The PLM shares its origin with different muscles such as the flexor digitorum superficialis muscle, the flexor carpi radialis muscle, and the flexor carpi ulnaris muscle [4].

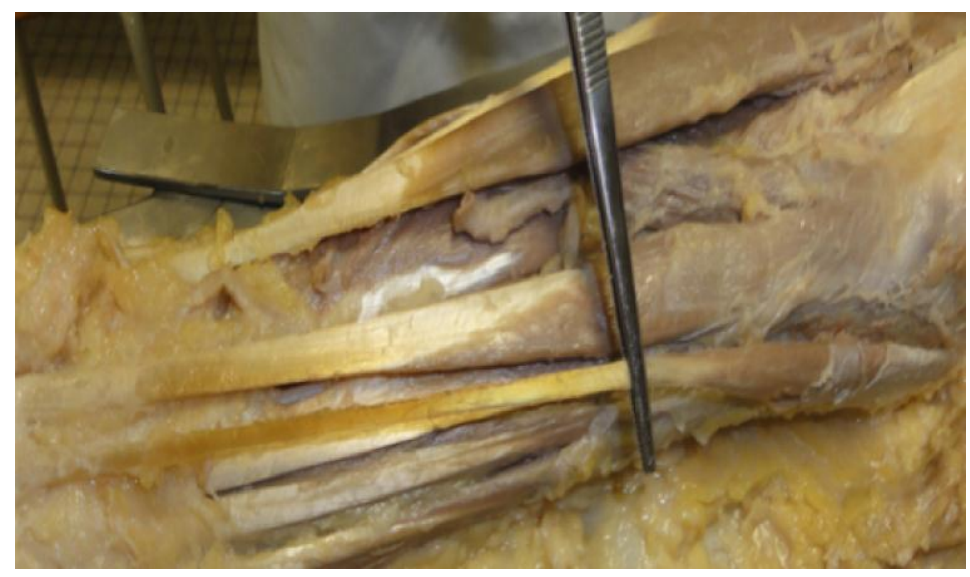

Figure 1. The palmaris longus muscle (PLM) with short proximal belly and longer distal tendon.

Anatomical variations of the muscle belly have been described by numerous reports. The many anatomical variants of the PLM are probably related to the human evolution [7]. The PLM can be hypertrophic, reversed, centrally placed, digastric, duplicated, or bifid. When the PLM is located deep to the ligamentum carpi transversum, it is also called a palmaris profundus muscle. This muscle can be categorized as a duplicated PLM [1]. A palmaris accesorius muscle can also occur and is recognized as a group of anomalies in origin and insertion and particular accessory insertion slips [7]. The PLM is vascularized by a small branch of the anterior ulnar recurrent artery and its innervation is derived from branches of the median nerve, which contains fibers from all five roots (C5-T1). The median nerve is derived from the medial and lateral cords of the brachial plexus [8].

Literature suggests that when the PLM is present, it may function as a weak wrist and elbow flexor [9]. Other functions of the PLM are assistance in carpo-metacarpal flexion and cupping of the palm [2], abduction of the thumb, stabilization of the superficial structures in the palm [10], and a tensor of the aponeurosis palmaris [6]. In subjects with an absent PLM, these functions also exist; therefore, it can be assumed that the presence of the PLM is of little overall value to hand function.

A lack of agreement is present in literature about the function of the PLM. This is why it is difficult to determine whether the PLM provides an advantage to handgrip in sport.

Fowlie et al. [11] determined the influence of the presence of the PLM in sports that require different sorts of handgrip. Characteristics of grips used for each of the participants' sport were compared. Tennis, badminton, and squash were defined as sustained dominant-sided cylindrical grip sports, while rowing and canoeing were considered as sustained two-handed cylindrical grip sports. In other sustained grip sports such as hockey and cricket, in which the stroke play is intermittent, the implement used is held throughout play. Golf was defined to be an intermittent grip sport because of the implement being released after stroke play. Fowlie et al. [11] reported that in both elite and non-elite athletes, the performance of the PLM was significantly higher in those who participated in sustained grip sports compared to intermittent grip sports. In elite athletes participating in dominant-handed and two-handed cylindrical grip sports, the presence of the PLM was greater on their dominant side compared with non-elite athletes participating in the same sports. Bilateral occurrence of the PLM was also higher in elite athletes [11].

Sebastin et al. [12] tested handgrip and pinch strength comparing subjects with and without PLM. These authors concluded that there was no significant difference in hand strength between the two groups. This was also determined in a pediatric population by Cetin et al [13]. As for grip strength, they found that the presence of the PLM did not create a difference in general. As for pinch strength, they 
concluded that the PLM increased pinch strength in the fourth and fifth finger of the hand, especially in the right hand. Based on these data, they suggested that the PLM could impact the opposition movement of the fingers [13].

Fleckenstein et al. [14] measured the contribution of the PLM to wrist flexion using magnetic resonance imaging before and after flexion exercises to determine muscle recruitment of wrist flexor muscle groups. Variations were determined by comparing the right and left forearm and the effect of slight $\left(15^{\circ}\right)$ pronation and supination of the wrist. Imaging after exercise showed focal regions of increased signal intensity, indicating relatively strong recruitment, most often in the whole muscle, although occasionally only in subvolumes of the muscles. Relatively strong recruitment of the flexor carpi radialis muscle was seen during pronation and/or use of the non-dominant side. They concluded that only seven of the eleven subjects recruited their PLM during isolated resisted wrist flexion with a weight of two kg [14].

The term proprioception, originally introduced by Sherrington, describes the sense of limb position and movements subserved by deep receptors in muscles, tendons, and joints [15], including capsular and ligamentous structures. The sensory end organs include the Pacinian receptors, Ruffini end receptors, Golgi tendon receptors, and free nerve endings. These aspects of afferent inputs are being increasingly studied in an attempt to describe and understand impairments, to improve rehabilitation effectiveness following trauma or surgery, and to prevent recurrent injury [16]. Different testing techniques have been developed to measure the three submodalities of proprioception: Joint position sense (JPS), kinesthesia (KIN), and sense of resistance [17]. The sources of proprioceptive information potentially include joint, muscle [18], and cutaneous mechanoreceptors [19]. Visual and auditory signals can provide additional cues to proprioception [20]. Each of these sensory receptors have been demonstrated to contribute to the sense of position or motion of a body part in space [21].

The methodologies used to gain information differ. In previous studies the modalities of proprioception have been measured by using dynamometers [22], electrogoniometers, potentiometers [23], electromagnetic sensors [21], and video digitization/analysis [24]. In this research we use the Flock of Birds tracking system, which captures the 3D position and orientation of a receiver with respect to a transmitter in six degrees of freedom, using pulsed magnetic fields.

In the present study further research has been conducted to determine whether presence of the PLM provides an advantage to handgrip in a sport such as tennis between elite tennis players and recreational athletes. Another aim of this study is to investigate the relationship between hand dominance and the performance of the PLM. The relation between the PLM and gender is also determined. The difference between presence or absence of the PLM and maximal grip strength and fatigue resistance is also a goal in this research. Furthermore maximal grip strength, fatigue resistance, and proprioceptive function of the hand and wrist between elite athletes with and without a PLM, is studied. Finally, this study tries to find out whether there is a difference between elite athletes and recreational athletes in the presence of the PLM, maximal grip strength, fatigue resistance, and proprioceptive function of the hand.

\section{Materials and Methods}

\subsection{Study Participants}

A total of 60 people participated in this study. The elite athletes group consisted of 30 healthy tennis players among which $23(76.7 \%)$ were male and seven $(23.3 \%)$ were female subjects. The elite athletes group included men with ranking A, B-15/4, B-15/2, or B-15/1, and women with ranking A, B-15/4, and B-15/2. Ranking is based on the tennis match results of certain competitions where they can deserve points. Tennisplayers are considered as an A player if they have an Association of Tennis Professionals (ATP)/Women's Tennis Association (WTA) ranking in the top 1000 (the first $5 \%$ of men, the first $10 \%$ of women). Ranking B-15/4 consists of tennisplayers with an ATP/WTA ranking above number 1000 , by men this means the following $10 \%$ of the tennisplayers, by women the following 
$15 \%$ of the players. B- $15 / 2$ players are being classified as the following $20 \%$ of these players by men and the following $20 \%$ by women. B- $15 / 1$ are the following $30 \%$ by men, and the following $25 \%$ by women. These athletes may also have reached this level in the past, up to a maximum ten years ago. The control group included 30 (23 male and 7 female) healthy recreational athletes who do not practice sports in which grip function is important such as tennis, badminton, squash, hockey, table tennis, etc.

Participants having a background of an injury or a surgical operation of the elbow or hand or having any pathology with a possible negative influence on the function of the hand, like carpal tunnel syndrome, diabetes, neurological disorder, or a self-reported history of inflammatory joint disease were excluded from the study.

The Human Ethics Committee of the Brussels Academic Hospital (UZ Brussel) approved the study on June 18, 2014 in accordance with the declaration of Helsinki of 1975. The project identification code of this study was B.U.N. 143201419488. Before participation in this research, all participants provided written informed consent. If the participant was younger than 18 years old, the informed consent was signed by the parents. The elite tennis players were recruited based on the club where they play tennis or through random contacts or friends. The control group was selected at random. Gender and age matching was attempted between elite sports and recreational athletes.

The measurements were organized at the tennis club of the players, at the researchers' place or at the Experimental Anatomy lab of the Vrije Universiteit Brussel (VUB) campus Jette. Measurements were performed from June to August 2014.

At first, personal data such as name, gender, age, hand dominance, sports, and ranking were registered. Then, the presence of the PLM, maximal grip strength, fatigue endurance, and proprioception were evaluated.

\subsection{Palmaris Longus Muscle}

Sebastin et al. [25] described six different techniques for examining the presence of the PLM in vivo (Figure 2). Initially, the subject was asked to perform the standard test on both sides. This was the most commonly used technique. If the palmaris longus tendon was not visualized or palpable, the second test was performed, and so on, until the last test. When the six techniques were performed without observation of a palmaris longus tendon, the presence of PLM was excluded. Tests were performed in the following order:

- Standard test (Schaeffer's test): opposition of the tip of the thumb to the tip of the little finger and then flexion of the wrist [26].

- Thompson's test: fist is made, then flexed, finally the thumb is opposed and flexed over the fingers [27].

- Mishra's test I: passive hyperextension of the metacarpophalangeal joints of all fingers, then the subject is asked to actively flex the wrist [28].

- Mishra's test II: abduction of the thumb against resistance with the wrist in slight palmar flexion [28].

- Pushpakumar's "two-finger sign" method: fully extension of the index and middle finger, flexion of the wrist and other fingers, finally the thumb is fully opposed and flexed [29].

- Gangata's test: manual isometric resistance against wrist flexion onto the palmar surface at the tips of the second to fifth metacarpals and, simultaneously, manual isometric resistance is applied against thumb abduction onto the lateral surface (where the palmar skin of the hand meets the dorsal skin) of the first metacarpophalangeal joint [5]. 


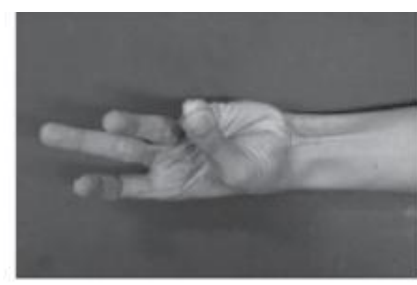

A

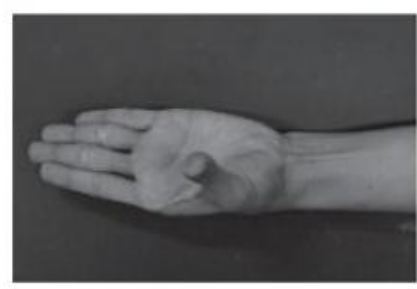

D

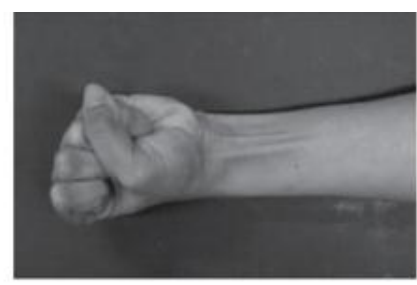

B

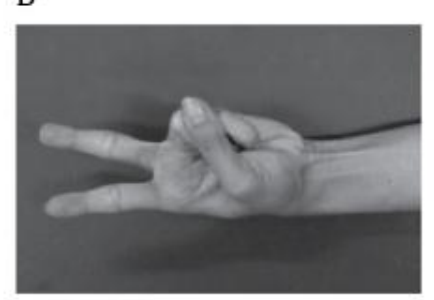

$\mathrm{E}$

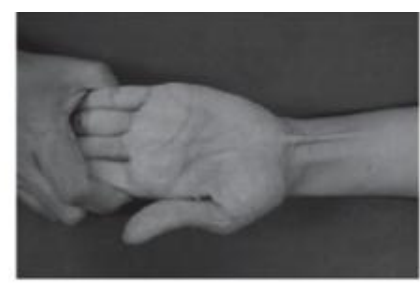

C

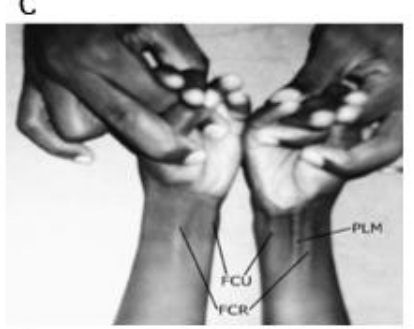

$\mathrm{F}$

Figure 2. Tests for detecting the presence of the PLM: (A) standard Schaeffer's test; (B) Thompson's test; (C) Mishra's test I; (D) Mishra's test II; (E) Pushpakumar's test; and (F) Gangata's test.

\subsection{Maximal Grip Strength and Fatigue Resistance}

Maximal grip strength and fatigue resistance were measured in both hands with an electronic hand dynamometer (CAMRY, model EH101, Zhongshan, China) (Figure 3) while the subject was seated on a chair. This device allowed continuous monitoring and recording of the pressure. The tests were executed with the shoulder in slight abduction and neutral rotation, the elbow flexed at $90^{\circ}$ and neutral position of the forearm and wrist. The upper arm and forearm could not touch the trunk. Then, the subject was asked to squeeze the handles towards each other as hard as possible. To ensure that maximal effort would be reached, participants were instructed to perform three sets with $20 \mathrm{~s}$ of rest between the sets, alternating the right and left hand, starting with the dominant hand. The highest of three attempts was described as the maximal grip strength. All pressure measurements were expressed in kilograms $(\mathrm{kg})$.

After this, the subject was again instructed to squeeze the handles towards each other as hard as possible and to maintain this maximal pressure until exhaustion. The time (in seconds) during which grip strength dropped to $50 \%$ of its maximum was noted as fatigue resistance [30].

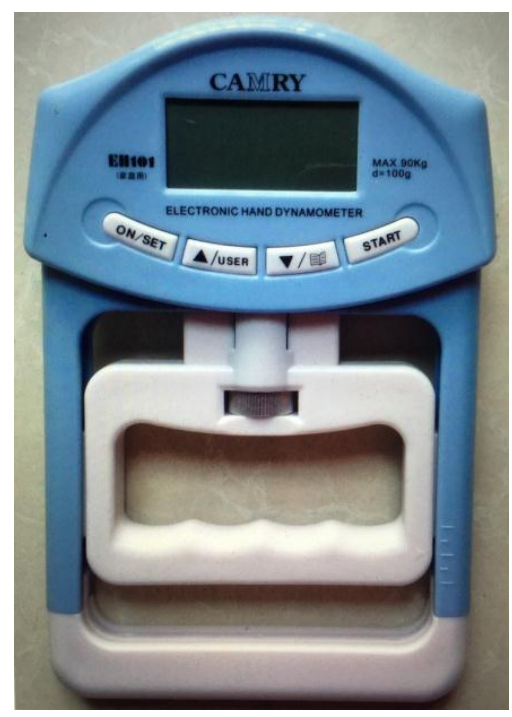

Figure 3. Electronic hand dynamometer (CAMRY, model EH101). 


\subsection{Proprioception}

Proprioception was defined as the cumulative neural input to the central nervous system from mechanoreceptors, which were located in the joint capsules, ligaments, muscles, tendons, and skin [31]. The ability to control the position of a joint was considered to be an aspect of proprioceptive functioning. Kinematics of the hand were registered by means of the Flock of Birds electromagnetic tracking system (Ascension Technologies, Shelburne, VT, USA) (Figure 4). This system was able to measure 3D positional and orientation data from multiple sensors. All ferromagnetic materials were removed from the evaluation area to avoid interference $[32,33]$. Therefore, the tests were executed on a wooden chair and table.

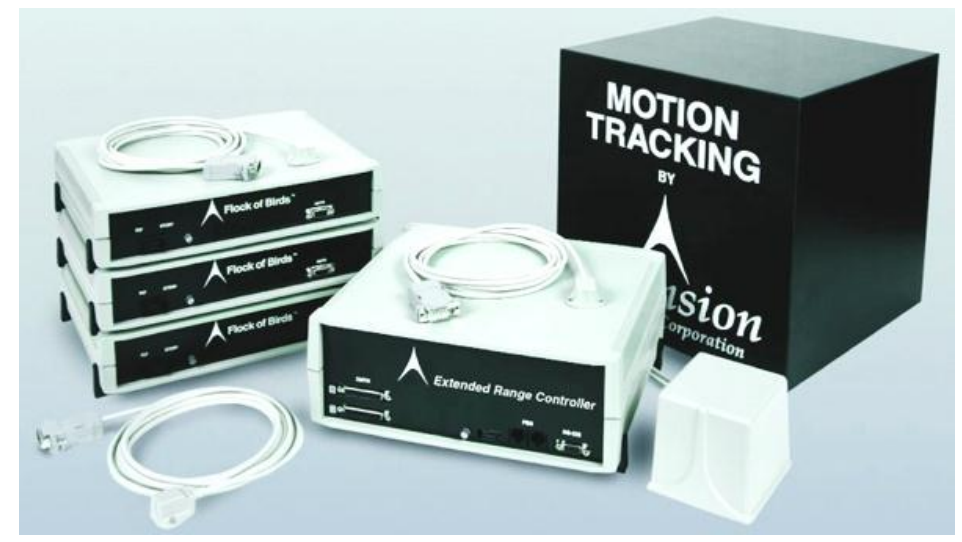

Figure 4. The Flock of Birds electromagnetic tracking system.

Each subject was seated in a chair with alternately one forearm placed in the arm supports. The subject's arm was positioned in forearm pronation and neutral wrist flexion/extension. A construction was made to ensure the forearm was fixated with a strap proximal of the processus styloideus radii and ulnae, and a strap distal of the olecranon, while solitary hand movements were performed. One sensor was attached proximal and in line with the dorsal aspect of the metacarpo-phalangeal III joint, in the middle between the distal end of metacarpo-phalangeal III and a transverse line between processus styloideus radii and ulnae. The other sensor was mounted on a stabile support on a hard foam mat as shown in Figure 5. The electromagnetic sensors were fixed with adhesive tape and velcro and registered angular displacement.

First, the JPS - the accuracy in which subjects can replicate a certain joint angle-was measured [17]. After starting in neutral position (fingers in line with the forearm), the subject was requested to move to palmar and dorsal flexion with the hand three times and, finally, try to replicate the initial neutral position. The joint position error between the initial position and the repositioning position was calculated. To eliminate visual cues the subject's view of the test joint was blocked.

In the second test kinesthesia (KIN) - the conscious awareness of position and movement in the joint, resulting from proprioceptive input to the central nervous system-was measured by performing a trajectory movement using a U-formed hard foam (Figure 6) [31]. The subject had to start in neutral position again in the U-formed hard foam, then move to palmar flexion and dorsal flexion until the fingers reached the foam while keeping the fingers in line with the forearm. This movement was repeated ten times with foam. Afterwards, the foam was removed and the subject had to perform the same movements three times, trying to reproduce, as accurately as possible, the previous range. In this test, the subject was also not allowed to look at the hands.

The third test consisted of movements, with and without feedback of the computer screen, which is called joint motion sense (JMS) in our study.

The participant had to start in neutral position, then move three times, first, 20 degrees to palmar flexion, and then 20 degrees to dorsal flexion, with feedback of the computer screen. Thereafter the 
same movement had to be performed three times, looking at the computer screen, but having the eyes closed without feedback.
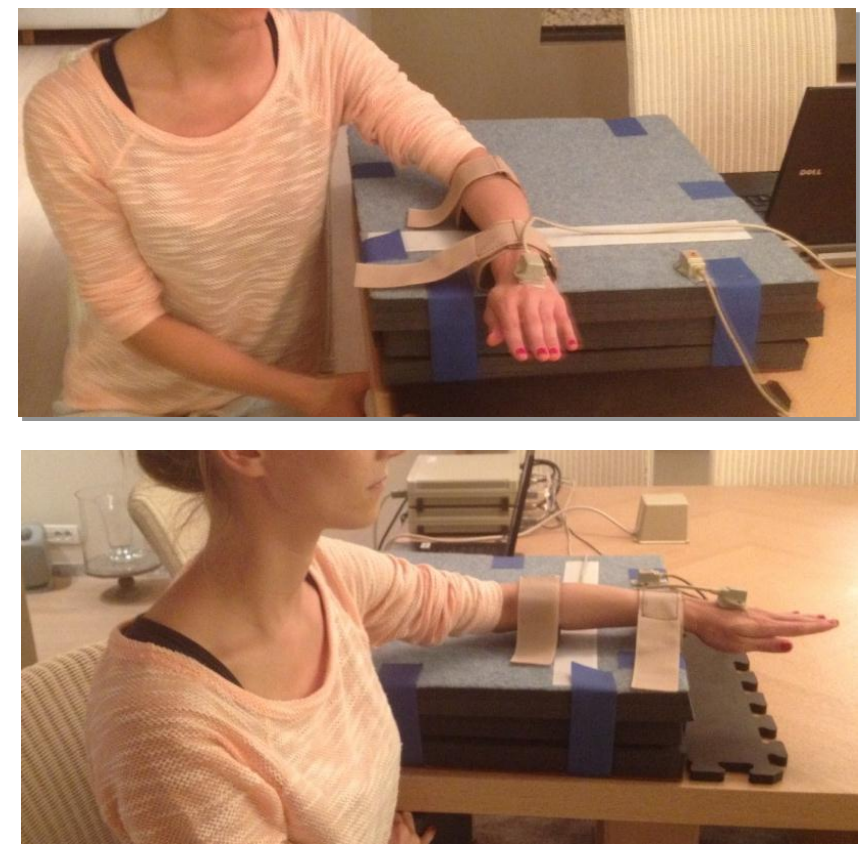

Figure 5. Experimental setup. Three tests were set up which can be regarded as specific for proprioceptive mechanisms.

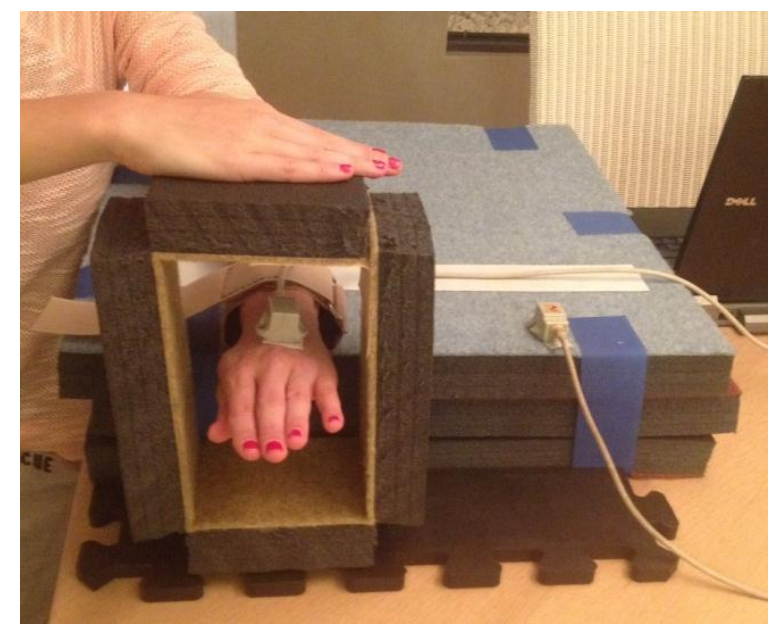

Figure 6. Measurement of kinesthesia with an U-formed hard foam.

\subsection{Data Analysis}

Data were collected in Microsoft Excel spreadsheets and kinematic processing for the proprioception components was conducted in a MathCAD 14 mathematical software routine. The range of motion was calculated and displayed in three axes $(X, Y, Z)$. The $Y$ coordinate presented the palmar and dorsal flexion of the hand, which could be seen as the main motion component. Other coupled motion components $(X$ and $Z$ ) were excluded from the analysis. JPS was calculated by comparing the position of the $Y$ coordinate with respect to the starting position, which was zero. KIN was measured by comparing the range of motion of the $Y$ coordinate of the movements with and without the foam. 
Calculation of the JMS was conducted by comparing the range of motion of the $\mathrm{Y}$ coordinate without feedback of the computer minus forty $\left(20^{\circ}\right.$ palmar and $20^{\circ}$ dorsal flexion). All of these outcomes were displayed in absolute values. Kinematic data were statistically analyzed using SPSS version 19 (IBM Corporation, Armonk, NY, USA), the statistical software program. Normality of data distribution was checked using the Kolmogorov-Smirnov test $(p<0.05)$.

The relationship between hand dominance and the performance of the PLM was determined by a Chi-square test. This test was also applied to analyze the correlation between the occurrence of the PLM and elite tennis players or recreational athletes.

Differences between subjects, controls, and subgroups (presence of the PLM, maximal grip strength, fatigue resistance, and proprioceptive function of the hand and wrist) were investigated using the analysis of covariance (ANCOVA). A value of $p<0.05$ was considered statistically significant.

\section{Results}

\subsection{Descriptive Statistics}

First, descriptive statistics were calculated for each testing (Table 1). Sixty subjects of Belgian descent were included in this study. The average age of the whole population is 22.3 years, ranging from 14 to 38 years old. The average age of the elite athletes was 22.6 years, ranging from 15 to 38 years old, while that of the control group was 22 years, ranging from 14 to 28 years old. The average grip strength on the dominant side was $48.41 \mathrm{~kg}$ and varied from 25 to $69.80 \mathrm{~kg}$, while on the non-dominant side it was $45.04 \mathrm{~kg}$, varying from 25.20 to $69.70 \mathrm{~kg}$. Fatigue resistance had a range from 11 to $85 \mathrm{~s}$ on the dominant side, and ranged from 8 to $89 \mathrm{~s}$ on the non-dominant side.

Table 1. Descriptive statistics.

\begin{tabular}{|c|c|c|c|c|c|c|c|c|c|c|c|c|}
\hline \multicolumn{2}{|c|}{ Parameters } & Age & $\begin{array}{c}\text { F } \\
\text { DOM }\end{array}$ & $\begin{array}{c}\text { F } \\
\text { NDOM }\end{array}$ & $\begin{array}{l}\text { FUH } \\
\text { DOM }\end{array}$ & $\begin{array}{c}\text { FUH } \\
\text { NDOM }\end{array}$ & $\begin{array}{c}\text { JPS } \\
\text { DOM }\end{array}$ & $\begin{array}{c}\text { JPS } \\
\text { NDOM }\end{array}$ & $\begin{array}{c}\text { KIN } \\
\text { DOM }\end{array}$ & $\begin{array}{c}\text { KIN } \\
\text { NDOM }\end{array}$ & $\begin{array}{l}\text { JMS } \\
\text { DOM }\end{array}$ & $\begin{array}{c}\text { JMS } \\
\text { NDOM }\end{array}$ \\
\hline \multicolumn{2}{|l|}{$\mathrm{N}$} & 60 & 60 & 60 & 60 & 60 & 60 & 60 & 60 & 60 & 60 & 60 \\
\hline \multicolumn{2}{|c|}{$\begin{array}{l}\text { Std. Error of } \\
\text { Mean }\end{array}$} & 0.56 & 1.52 & 1.45 & 2.31 & 2.11 & 0.68 & 0.55 & 1.10 & 1.20 & 1.05 & 0.89 \\
\hline \multicolumn{2}{|c|}{ Median } & 22.00 & 51.15 & 45.15 & 35.50 & 34.50 & 2.68 & 4.61 & 8.59 & 7.57 & 7.12 & 10.52 \\
\hline \multicolumn{2}{|c|}{ Variance } & 19.41 & 140.14 & 126.89 & 321.54 & 267.63 & 27.83 & 18.47 & 72.89 & 86.41 & 67.11 & 47.86 \\
\hline \multicolumn{2}{|c|}{ Range } & 24 & 44.80 & 44.50 & 74 & 81 & 22.33 & 18.36 & 31.23 & 39.99 & 35.90 & 27.45 \\
\hline \multicolumn{2}{|c|}{ Minimum } & 14 & 25.00 & 25.20 & 11 & 8 & 0.02 & 0.04 & 0.25 & 0.23 & 0.10 & 0.05 \\
\hline \multicolumn{2}{|c|}{ Maximum } & 38 & 69.80 & 69.70 & 85 & 89 & 22.35 & 18.40 & 31.48 & 40.22 & 36.00 & 27.50 \\
\hline \multirow[b]{2}{*}{ Percentiles } & 25 & 18.25 & 38.92 & 36.57 & 26.25 & 24.00 & 1.31 & 2.31 & 4.60 & 3.70 & 2.30 & 5.11 \\
\hline & 50 & 22.00 & 51.15 & 45.15 & 35.50 & 34.50 & 2.68 & 4.61 & 8.59 & 7.57 & 7.12 & 10.52 \\
\hline
\end{tabular}

a: multiple modes exist. The smallest value is shown. N: number of participants; DOM: dominant side; NDOM: non-dominant side; F: maximal grip strength; FUH: fatigue resistance; JPS: joint position sense; KIN: kinesthesia; JMS: joint motion sense.

\subsection{Palmaris Longus Muscle—Hand Dominance}

In this study population of 60 people, 50 subjects (83.3\%) were right-handed and $10(16.6 \%)$ were left-handed.

In right-handed participants, five people (10\%) occured without a PLM, three $(6.0 \%)$ with a unilateral PLM on the right side, one (2.0\%) with a unilateral PLM on the left side, and $41(82.0 \%)$ with a PLM on both sides. In the left-handed group, one person (10.0\%) had a unilateral PLM on the right side, another person $(10.0 \%)$ had a unilateral PLM on the left side, eight (80\%) people had a PLM on both sides, while nobody occured without a PLM (Table 2). The relationship between hand dominance and the presence of the PLM was not significant $(p=0.423)$. 
Table 2. The relationship between hand dominance and the PLM.

\begin{tabular}{cccccc}
\hline Hand Dominance & No PLM & Unilateral PLM Right & Unilateral PLM Left & Bilateral PLM & Total \\
\hline Right-handed & $5(10.0 \%)$ & $3(6.0 \%)$ & $1(2.0 \%)$ & $41(82.0 \%)$ & $50(100 \%)$ \\
Left-handed & $0(0.0 \%)$ & $1(10.0 \%)$ & $1(10.0 \%)$ & $8(80 \%)$ & $10(100 \%)$ \\
\hline
\end{tabular}

Values refer to number of participants with or without a PLM tested by the six tests described in Figure 2. PLM: palmaris longus muscle.

\subsection{Palmaris Longus Muscle-Gender}

Table 3 represents the relationship between gender and the presence of the PLM. $80.4 \%$ of men and $85.7 \%$ of women had a bilateral PLM. Five $(10.9 \%)$ men had no PLM, three $(6.5 \%)$ had a unilateral PLM on the right side, and one (2.2\%) man had a unilateral PLM on the left side. While in the female group one (7.1\%) woman had a right unilateral PLM, and one (7.1\%) woman had a unilateral PLM on the left side. No significant relationships between the presence of the PLM and gender were found $(p=0.501)$.

Table 3. The relationship between gender and the PLM.

\begin{tabular}{cccccc}
\hline Gender & No PLM & Unilateral PLM Right & Unilateral PLM Left & Bilateral PLM & Total \\
\hline Men & $5(10.9 \%)$ & $3(6.5 \%)$ & $1(2.2 \%)$ & $37(80.4 \%)$ & $46(100 \%)$ \\
Women & $0(0.0 \%)$ & $1(7.1 \%)$ & $1(7.1 \%)$ & $12(85.7 \%)$ & $14(100 \%)$ \\
\hline
\end{tabular}

All values were expressed in number of participants with and without a PLM tested by the six tests described in Figure 2. PLM: palmaris longus muscle.

\subsection{Palmaris Longus Muscle-Elite Tennis Players and Recreational Athletes}

The group of elite tennis players consisted of $86.7 \%$ with a PLM on both sides, $6.7 \%$ had a unilateral PLM on the right side, 3.3\% had one on the left side, and 3.3\% had no PLM at all. In the control group, also named the recreational or non-elite athletes, $76.7 \%$ had a bilateral PLM, $13.3 \%$ had no PLM, 6.7\% had a PLM on the right side, and 3.3\% showed a PLM on the left side (Table 4). No significant relation between elite and non-elite athletes and the presence of the PLM was established $(p=0.576)$.

Table 4. The relationship between elite tennis players and recreational athletes and the presence of the PLM.

\begin{tabular}{cccccc}
\hline Participant Group & No PLM & Unilateral PLM Right & Unilateral PLM Left & Bilateral PLM & Total \\
\hline Elite tennis players & $1(3.3 \%)$ & $2(6.7 \%)$ & $1(3.3 \%)$ & $26(86.7 \%)$ & $30(100 \%)$ \\
Non elite athletes & $4(13.3 \%)$ & $2(6.7 \%)$ & $1(3.3 \%)$ & $23(76.7 \%)$ & $30(100 \%)$ \\
\hline
\end{tabular}

Values refer to number of participants with and without a PLM tested by the six tests described in Figure 2 . PLM: palmaris Longus muscle.

\subsection{Elite Tennis Players and Recreational Athletes-Maximal Grip Strength and Fatigue Resistance}

For maximal grip strength on the dominant side, significant differences were found for age $(p=0.043)$ and gender $(p=0.000)$. On the non-dominant side there was only a significant difference for gender $(p=0.000)$. Fatigue resistance on the dominant side presented no significant differences, but on the non-dominant side it was significant for the elite group and non-elite group $(p=0.009)$ (Table 5).

\subsection{Elite Tennis Players and Recreational Athletes-Proprioceptive Function}

The relationship of the proprioceptive function with age, gender, elite and non-elite athletes, and the presence of the PLM, was only significant between KIN on the non-dominant side and age $(p=0.019)$ and between JMS on the non-dominant side and gender $(p=0.023)$ (Table 6). 
Table 5. The level of significance in the relationship between maximal grip strength, fatigue resistance and subgroups on the dominant and non-dominant side in elite tennis players and recreational athletes.

\begin{tabular}{ccccc}
\hline Traits & $\begin{array}{c}\text { Maximal Grip } \\
\text { Strength Dominant }\end{array}$ & $\begin{array}{c}\text { Maximal Grip } \\
\text { Strength } \\
\text { Non-Dominant }\end{array}$ & $\begin{array}{c}\text { Fatigue Resistance } \\
\text { Dominant }\end{array}$ & $\begin{array}{c}\text { Fatigue Resistance } \\
\text { Non-Dominant }\end{array}$ \\
\hline Age & $0.043^{*}$ & 0.242 & 0.442 & 0.390 \\
Gender & $0.000^{*}$ & $0.000^{*}$ & 0.414 & 0.285 \\
Elite-non Elite & 0.065 & 0.054 & 0.203 & $0.009 *$ \\
PLM & 0.201 & 0.859 & 0.299 & 0.600 \\
\hline
\end{tabular}

Maximal grip strength (expressed in kilograms) and fatigue resistance (expressed in seconds) were measured by an electronic hand dynamometer (Figure 3). Values refer to the level of significance. *: significant difference $(p<0.05) ;$ PLM: palmaris longus muscle.

\subsection{Subgroup Elite Tennis Players-Maximal Grip Strength and Fatigue Resistance}

When only looking at the subgroup of elite tennis players, maximal grip strength on the dominant and non-dominant side was only significant with gender $(p=0,000)$. The other variables showed no significant differences (Table 7).

\subsection{Subgroup Elite Tennis Players-Proprioceptive Function}

As for the proprioceptive function in the subgroup of elite tennis players, the presence of the PLM, age, and gender did not reflect any significant differences (Table 8).

Table 6. The level of significance in the relationship between proprioceptive function (JPS, KIN, JMS) and subgroups on the dominant and non-dominant side in elite tennis players and recreational athletes.

\begin{tabular}{ccccccc}
\hline Traits & $\begin{array}{c}\text { JPS } \\
\text { Dominant }\end{array}$ & $\begin{array}{c}\text { JPS } \\
\text { Non-Dominant }\end{array}$ & $\begin{array}{c}\text { KIN } \\
\text { Dominant }\end{array}$ & $\begin{array}{c}\text { KIN } \\
\text { Non-Dominant }\end{array}$ & $\begin{array}{c}\text { JMS } \\
\text { Dominant }\end{array}$ & $\begin{array}{c}\text { JMS } \\
\text { Non-Dominant }\end{array}$ \\
\hline $\begin{array}{c}\text { Age } \\
\text { Dender }\end{array}$ & 0.102 & 0.292 & 0,383 & $0.019 *$ & 0.157 & 0.711 \\
Elite-non & 0.821 & 0.289 & 0.242 & 0.465 & 0.623 & $0.023 *$ \\
elite & 0.537 & 0.125 & 0.721 & 0.485 & 0.545 & 0.296 \\
PLM & 0.817 & 0.926 & 0.364 & 0.412 & 0.142 & 0.903 \\
\hline
\end{tabular}

JPS, KIN and JMS were measured by the Flock of Birds electromagnetic tracking system. JPS described the replication of a certain joint angle, KIN was measured by performing a trajectory movement using a U-formed hard foam and JMS consisted of movements with and without feedback of the computer. All values refer to the level of significance. *: significant difference $(p<0.05)$; PLM: palmaris longus muscle; JPS: joint position sense; KIN: kinesthesia; JMS: joint motion sense.

Table 7. The level of significance in the relationship between maximal grip strength, fatigue resistance, and subgroups on the dominant and non-dominant side in elite tennis players.

\begin{tabular}{ccccc}
\hline Traits & $\begin{array}{c}\text { Maximal Grip } \\
\text { Strength Dominant }\end{array}$ & $\begin{array}{c}\text { Maximal Grip Strength } \\
\text { Non-Dominant }\end{array}$ & $\begin{array}{c}\text { Fatigue Resistance } \\
\text { Dominant }\end{array}$ & $\begin{array}{c}\text { Fatigue Resistance } \\
\text { Non-Dominant }\end{array}$ \\
\hline Age & 0.930 & 0.961 & 0.387 & 0.095 \\
Gender & $0.000^{*}$ & $0.000^{*}$ & 0.671 & 0.466 \\
PLM & 0.136 & 0.985 & 0.304 & 0.088 \\
\hline
\end{tabular}

Maximal grip strength (expressed in kilograms) and fatigue resistance (expressed in seconds) were measured by an electronic hand dynamometer (Figure 3). Values refer to the level of significance. *: significant difference $(p<0.05)$; PLM: palmaris longus muscle. 
Table 8. The level of significance of the relationship between proprioceptive function (JPS, KIN, JMS) and subgroups on the dominant and non-dominant side in elite tennis players.

\begin{tabular}{ccccccc}
\hline Traits & $\begin{array}{c}\text { JPS } \\
\text { Dominant }\end{array}$ & $\begin{array}{c}\text { JPS } \\
\text { Non-Dominant }\end{array}$ & $\begin{array}{c}\text { KIN } \\
\text { Dominant }\end{array}$ & $\begin{array}{c}\text { KIN } \\
\text { Non-Dominant }\end{array}$ & $\begin{array}{c}\text { JMS } \\
\text { Dominant }\end{array}$ & $\begin{array}{c}\text { JMS } \\
\text { Non-Dominant }\end{array}$ \\
\hline Age & 0.298 & 0.203 & 0.530 & 0.170 & 0.527 & 0.941 \\
Gender & 0.995 & 0.355 & 0.676 & 0.060 & 0.893 & 0.605 \\
PLM & 0.749 & 0.153 & 0.755 & 0.427 & 0.347 & 0.646 \\
\hline
\end{tabular}

JPS, KIN, and JMS were measured by the Flock of Birds electromagnetic tracking system. JPS described the replication of a certain joint angle, KIN was measured by performing a trajectory movement using a U-formed hard foam, and JMS consisted of movements with and without feedback of the computer. All values refer to the level of significance. PLM: palmaris longus muscle; JPS: joint position sense; KIN: kinesthesia; JMS: joint motion sense.

\section{Discussion}

The PLM agenesis has been variously reported to be from 3.0\% in black people to $63.9 \%$ in Turkish people. The prevalence of absence of the PLM in the Caucasian group was found to be $22.4 \%$, including unilateral and bilateral agenesis [2]. It is important to be aware of the variability in prevalence of this muscle related to the population or ethnic group.

The prevalence of agenesis was found to be significantly more common on the left side [3,34]. This corresponds to our study, in which four subjects had a unilateral PLM on the right side while only two subjects showed a unilateral PLM on the left side.

The incidence of left-handedness is less common than right hand dominance. About $8 \%-15 \%$ of the general population is left-handed [35]. In this study, $16.6 \%$ of the participants were left-handed, which corresponds to the general population. Eric et al. [36] reported in his study that a right-sided absence was more common in left-handed persons while the left-sided absence was more common in right-handed persons. Unilateral tendon absence was more common in the non-dominant hand. This study included 542 subjects. Due to the size of the study population, these results were very reliable. Our study (Table 1) also showed that in the right-handed participants left-sided absence was more common. In left-handed dominance, however, the absence of the PLM was the same on both sides. Although in agreement with Eric et al., due to the small size of the study population, this conclusion is not significant and cannot be generalized [36].

In general, most people have a bilateral PLM, second comes bilateral absence, unilateral absence on the right is next, and unilateral absence on the left is less common. This was the case in our study and was also reported by Eric et al. [36].

Other studies however reported another descending order: bilateral present, bilateral agenesis, left agenesis, and right agenesis $[1,3,26,37]$. Finally Soltani et al. found that bilateral presence of the PLM occurred the most, followed by unilaterally absence of the PLM while bilaterally absence of the PLM occurred less [34].

Thompson et al. reported that men were more prone to have a bilateral and unilateral absence of the PLM, but this was not statistically significant [27]; other authors reported the incidence of agenesis to be higher in women $[1,26,34]$.

The study of Kapoor et al. [3] also described that men were more common to have a unilateral agenesis and women to have a bilateral agenesis. This difference was statistically significant. This is in contrast with our findings in which women were more likely to have a unilateral PLM while men were more likely to have no PLM at all, but there was, however, no significant difference. According to Eric et al., the middle-age and young group showed a lower rate of the PLM presence as compared to the old group, although this difference was not significant [36,38]. In comparison, Venter et al. reported no obvious trend in the PLM in the various age groups that would indicate phylogenetic degeneration of the PLM [39].

The frequent use of the PLM in surgery begged the question whether the removal of this muscle would affect the hand function [13]. The PLM is often used for reconstructive surgery because it fulfilles 
the criteria of length, thickness and availability without producing a deformity [40]. Verdan [41] suggested that a divided PLM did not need to be repaired because of its small importance, while Sebastin et al. [12] believed that repair of a divided PLM should always be done, even if it were the only divided tendon, as a repaired PLM was considered to protect the median nerve, which is necessary, especially in patients with self-mutilation. Repair of the PLM provided recovery of the function of this muscle, and saved it as a tendon graft for later use [12].

The presence of the PLM, on the other hand, was correlated with a significant increase in risk of developing Dupuytren's disease [42], a common acquired pathological condition with fibrous degeneration of the superficial longitudinal layer of the aponeurosis and the retinacula cutis. The deep transverse layer seems to be only involved in late stages of the disease [43].

Fowlie et al. suggested that the presence of the PLM could provide a favorable contribution to sustained grip sports and cylindrical grip sports that require a higher level of skill [11]. In athletes who participate in a sustained or cylindrical grip sport, the PLM may provide a larger pool of muscle fibers that can be recruited for strength and endurance, or a greater pool of proprioceptors that can contribute to superior grip precision. To maintain a stable grip during sustained grip sports, a submaximal isometric co-contraction of carpo-metacarpal flexors and finger flexors is required. To stabilize the wrist in a specific position, co-contraction of wrist flexors and extensors is also likely to occur [11]. Chow et al. and Wei et al. supported the theory that there is electromyographic activity of wrist flexors and extensors during backhand and forehand volleys in tennis [44,45]. Chow et al. [44] studied the muscle activation characteristics of a tennis volley under varying ball speed and location conditions. This was determined by surface electromyography techniques which could not differentiate between individual muscles but only activation of the flexors and extensors in general. In all subjects, muscle activity increased when the ball speed was higher. This suggests that the grip force and wrist stiffness increased as ball speed increased. Assuming that a quicker racket movement is required in fast speed trials, an expansion in forearm muscle activity may be required as the speed of racket movement expanses [44], which suggests that elite tennis players require more muscle activity because of higher ball speed.

Some authors have found that absence of the PLM was not significantly correlated with decreased grip or pinch strength measurements [12,13]. Cetin et al. [13] on the other hand, reported that having a PLM increased the pinch strength in the fourth and fifth finger of the hand, especially in the right hand. For the pinch strength of the second finger, no significant difference was found. The third finger of the right hand showed only in girls of 6-7 years old a significant correlation with the absence of the PLM, but no relation was found for the left hand. In addition, this muscle may impact the opposition movement of the fingers [13]. These findings suggest that the PLM may not be as redundant as current opinion indicates, although we have to keep in mind that this study only included girls and boys with ages of 6-11 years old.

Petersen et al. described a difference in grip strength between the dominant and non-dominant hand in left-handed subjects ranging from 0 to $5 \%$, while in right-handed subjects the dominant hand is generally accepted to be $10 \%$ stronger than the non-dominant hand [46]. In our study this was not always the case, as a lot of variations occurred between grip strength of the dominant and non-dominant hand.

The grip strength could also be influenced by the occupation of the subjects. It is logical that manual workers have a greater grip strength compared to sedentary workers and that people who use their hand function a lot may have a better grip strength. Another thing that has to be kept in mind is that we did not consider differences in body mass of our participants.

Unfortunately, kinesthesia and proprioception are used interchangeably in literature, which leads to confusion of these terms. Proprioception refers to the general central nervous system, which describes afferent information arising from internal peripheral areas of the body that have a contribution to postural control, joint stability and several conscious sensations [47]. The advantage of registering active movement with the Flock of Birds electromagnetic tracking system is that subjects 
have free, unrestricted movement, unlike in the proprioception testing device, where they are limited to one degree of freedom. This is important for the wrist, because natural movement patterns involve multiplanar motions [17].

As far as we know, this is the first study that measured the wrist proprioception in relation with the presence of the PLM and in relation with an elite sports population.

In the present study there is no significant correlation between the proprioceptive function and subgroups. The only significant relation is between kinesthesia on the non-dominant side and age, and between joint motion sense on the non-dominant side and gender, but this could be due to coincidence. No previous studies were found about the relationship between the proprioceptive function and gender, age, the PLM, or elite and non-elite athletes. This topic requires more research with a larger population to increase the validity.

The advantage of this measurement system for wrist joint proprioception allows the researcher to decrease extraneous influences that may affect joint position sense awareness and, therefore, improve the knowledge of the mechanisms underlying kinesthesia and proprioception. Gay et al. [16] approved, with an experimental setup similar to the one used in our study, that the measurements were repeatable and appeared to be equally or more accurate than other methods previously employed to measure wrist and hand range of motion. The difference with our study was, however, that subjects were tested in an active as well as in a passive condition [16]. According to Voight et al., subjects replicated movements more accurately after active target presentation than after passive [19], while other authors found no difference between active and passive repositioning [21,48].

Proprioceptive information plays an important role in joint stabilization. The stability of motion or changes in motion sequence may be an expression of altered or disturbed proprioception. According to Voight et al. [19] proprioception of the glenohumeral joint is significantly altered in the presence of shoulder muscle fatigue. They measured active and passive repositioning of the upper extremity accurately following a fatigue protocol [19]. With respect to this theory, it has to be kept in mind that some of the elite tennis players conducted the proprioceptive tests after warming-up or after having played a tennis game.

Concerning the absence of a significant difference in strength and proprioception of the wrist between subjects with and without a PLM, it is possible that its functional ability has been taken over by the other forearm flexors in subjects where it is absent.

Furthermore, absence may not necessarily be the same as loss, particularly in patients with pathology involving the other wrist flexors or thumb abductors. In such cases, removal of the PLM may further weaken the already damaged hand.

One of the limitations of the study was that we did not measure the pinch strength or thumb abduction. Some studies have reported the importance of the opposition movement and thumb abduction of the PLM, which is why these parameters might better be included in further investigations. It was difficult to measure the functional contribution of the PLM separate from the other wrist flexors and thumb abductors. We used grip strength for measuring the hand function because it provides us with quantitative evidence and is easily understood by patients. The present study was also limited in the study population that was too small because of its strong selection criteria to participate. Some of the subjects were still on high school or university, which means that they probably use their finger muscles for handwriting much more than adults. This could be of influence on the data. In order to determine whether the removal of the PLM results in change in grip and pinch strength of these patients, it is necessary to perform these tests before and after surgical removal of this muscle.

The ideal sample for this study would be a group of patients in whom the PLM has been used for reconstruction surgery in another region than the wrist. A pre- and post-operative assessment of grip and pinch strength could reflect the functional loss due to removal of the PLM. However, it is extremely difficult to get a large population of such patients.

The strength of this study was that it demonstrated the feasibility of the test protocol in terms of execution and that it examined more aspects of proprioception than most previous studies did. 
We have to keep in mind that a weakly-developed or anomalous PLM may have been mistaken as absent. However, a comparison between cadaveric and in vivo studies showed no significant difference in prevalence [2]. Radiological investigation such as an ultrasonography or magnetic resonance imaging would be a sure way of detecting even an anomalous tendon, but the performance of such an investigation in a large population might render the protocol less feasible.

\section{Conclusions}

In this study, the results indicate that the bilateral presence of the PLM is the most common variation, compared to absence of PLM. After that, unilateral PLM on the right side and unilateral PLM on the left side occurs less frequently. More elite tennis players have a bilateral PLM compared to the non-elite athletes, but this difference was not significant.

The results of the present study demonstrate significant differences for maximal grip strength on the dominant side for age and gender and on the non-dominant side only for gender. Fatigue resistance on the dominant side presented no correlations, but on the non-dominant side it was significantly different for the elite and non-elite groups. The relationship of the proprioceptive function with age, gender, elite, and non-elite athletes and the presence of the PLM, showed only a significant difference between KIN on the non-dominant side and age, and between JMS on the non-dominant side and gender.

Based on these findings, absence of the PLM does not seem to be correlated with decreased grip strength, fatigue resistance, or proprioceptive measurements.

Further research with larger population is needed to confirm that the absence of the palmaris longus muscle is not disadvantageous in the handgrip of elite tennis players.

Acknowledgments: We would like to thank Marie-Josée Dujardin for her English revision. We also would like to express my thanks to Bram Sorel and Bram Van Laere for sharing their knowledge on the subject and helped me out by taking a picture of anatomical dissection presence of the PLM.

Author Contributions: Julie Vercruyssen and Erik Cattrysse have made substantial intellectual contributions to the conception and design of the study. Julie Vercruyssen has performed and written the study. Erik Cattrysse and Aldo Scafoglieri have contributed to the evaluation of the data and revised and approved the manuscript. All authors have approved the final version submitted.

Conflicts of Interest: The authors declare no conflict of interest.

\section{References}

1. Reimann, A.; Dasseler, E.; Anson, B.; Beaton, L. The Palmaris longus muscle and tendon. A study of 1600 extremities. Anat. Rec. 1944, 89, 495-505. [CrossRef]

2. Sebastin, S.; Puhaindran, M.; Lim, A.; Lim, I.; Bee, W. The prevalence or absence of the Palmaris longus-A study in a Chinese population and a review of the literature. J. Hand Surg. Br. 2005, 30, 525-527. [CrossRef] [PubMed]

3. Kapoor, S.K.; Tiwari, A.; Kumar, A.; Bhatia, R.; Tantuway, V.; Kapoor, S. Clinical relevance of Palmaris longus agenesis: Common anatomical aberration. Anat. Sci. Int. 2008, 83, 45-48. [CrossRef] [PubMed]

4. Stecco, C.; Lancerotto, L.; Porzionato, A.; Macchi, V.; Tiengo, C.; Parenti, A.; Sanudo, J.; de Caro, R. The palmaris longus and its relations with the antebrachial fascia and the palmar aponeurosis. Clin. Anat. 2009, 22, 221-229. [CrossRef] [PubMed]

5. Gangata, H. The clinical surface anatomy anomalies of the Palmaris longus muscle in the Black African population of Zimbabwe and a proposed new testing technique. Clin. Anat. 2009, 22, 230-235. [CrossRef] [PubMed]

6. Schünke, M.; Schulte, E.; Schumacher, U. Prometheus; Bohn Stafleu van Loghum: Houten, Utrecht, Nederland, 2005.

7. De Smedt, L. Median and ulnar nerve compression at the wrist caused by anomalous muscles. Acta Orthop. Belgica. 2002, 68, 431-438.

8. Park, M.J.; Namdari, S.; Yao, J. Anatomic variations of the Palmaris longus muscle. Am. J. Orthop. 2010, 39, 89-94. [PubMed] 
9. Sobotta, J.; Becher, H. Atlas of Human Anatomy. In Regions, Skeleton, Ligaments, Joints and Muscles, 9th ed.; Ferner, H., Staubesand, J., Eds.; Urban and Schwarzenberg: München, Germany, 1975; Volume 1, pp. 193-198.

10. Fahrer, M. Proceedings: The role of the Palmaris longus muscle in the abduction of the thumb. J. Anat. 1973, 116, 476. [PubMed]

11. Fowlie, C.; Fuller, C.; Pratten, M.K. Assessment of the presence/absence of the palmaris longus muscle in different sports, and elite and non-elite sport populations. Physiotherapy 2012, 98, 138-142. [CrossRef] [PubMed]

12. Sebastin, S.J.; Lim, A.Y.; Wong, T.C.; Methil, B.V. Does absence of the Palmaris longus affect grip and pinch strength? J. Hand Surg. Br. 2005, 30, 406-408. [CrossRef] [PubMed]

13. Cetin, A.; Genc, M.; Sevil, S.; Coban, Y.K. Prevalence of the Palmaris longus muscle and its relationship with grip and pinch strength: A study in a Turkish pediatric population. Hand (NY). 2013, 8, 215-220. [CrossRef] [PubMed]

14. Fleckenstein, J.; Watumull, D.; Bertocci, L.; Nurenberg, P.; Peshock, R.; Payne, J.; Haller, R.G. Muscle recruitment variations during wrist flexion exercise: MR evaluation. J. Comput. Assist. Tomogr. 1994, 18, 449-453. [CrossRef] [PubMed]

15. Sherrington, C.S. On the proprioceptive system, especially in its reflex aspects. Brain 1906, $29,467-482$. [CrossRef]

16. Gay, A.; Parratte, S.; Salazard, B.; Gulnard, D.; Pham, T.; Legre, R.; Roll, J.P. Proprioceptive feedback enhancement induced by vibratory stimulation in complex regional pain syndrome type 1: An open comparative pilot study in 11 patients. Joint Bone Spine 2007, 74, 461-466. [CrossRef] [PubMed]

17. Riemann, B.L.; Myers, J.B.; Lephart, S.M. Sensorimotor system measurement techniques. J. Athl. Train. 2002, 37, 85-98. [PubMed]

18. Gandevia, S.C.; Burke, D. Does the nervous system depend on kinesthetic information to control natural limb movements? Behav. Brain Sci. 1992, 15, 614-632.

19. Voight, M.L.; Hardin, J.A.; Blackburn, T.A.; Tippett, S.; Canner, G.C. The effects of muscle fatigue on and the relationship of arm dominance to shoulder proprioception. J. Orthop. Sports Phys. Ther. 1996, 23, 348-352. [CrossRef] [PubMed]

20. Brindle, T.; Uhl, T.; Nitz, A.; Shapiro, R. Motor control of shoulder proprioception at various speeds. Phys. Ther. 2003, 23, 109-117.

21. Lönn, J.; Crenshaw, A.G.; Djupsjöbacka, M.; Pedersen, J.; Johansson, H. Position sense testing: Influence of starting position and type of displacement. Arch. Phys. Med. Rehabil. 2000, 81, 592-597. [CrossRef]

22. Janwantanakul, P.; Magarey, M.; Jones, M.; Dansie, B. Variation in shoulder position sense at mid and extreme range of motion. Arch. Phys. Med. Rehabil. 2001, 82, 840-844. [CrossRef] [PubMed]

23. Cash, R.; Gonzalez, M.; Garst, J.; Barmada, R.; Stern, S. Proprioception after arthroplasty: Role of the posterior cruciate ligament. Clin. Orthop. Relat. Res. 1996, 331, 172-178. [CrossRef] [PubMed]

24. Baker, V.; Bennell, K.; Stillman, B.; Cowan, S.; Crossley, K. Abnormal knee joint position sense in individuals with patellofemoral pain syndrome. J. Orthop. Res. 2001, 20, 208-214. [CrossRef]

25. Sebastin, S.J.; Lim, A.Y. Clinical assessment of absence of the palmaris longus and its association with other anatomical anomalies-A chinese population study. Ann. Acad. Med. Singapore 2006, 35, 249-253. [PubMed]

26. Schaeffer, J.P. On the variations of the Palmaris longus muscle. Anat. Rec. 1909, 3, 275-278.

27. Thompson, J.W.; McBatts, J.; Danforth, C.H. Hereditary and racial variations in the musculus Palmaris longus. Am. J. Phys. Anthrop. 1921, 4, 205-220. [CrossRef]

28. Mishra, S. Alternative tests in demonstrating the presence of Palmaris longus. Indian J. Plast. Surg. 2001, $34,12$.

29. Pushpakumar, S.B.; Hanson, R.P.; Carroll, S. The 'two finger' sign. Clinical examination of Palmaris longus (PL) tendon. Br. J. Plast. Surg. 2004, 57, 184-185. [CrossRef] [PubMed]

30. Bautmans, I.; Onyema, O.; van Puyvelde, K.; Pleck, S.; Mets, T. Grip work estimation during sustained maximal contraction: Validity and relationship with dependency and inflammation in elderly persons. J. Nutr. Health Aging. 2011, 15, 731-736. [CrossRef] [PubMed]

31. Wilkerson, G.B.; Nitz, A.J. Dynamic ankle stability: Mechanical and neuromuscular interrelationships. J. Sport Rehab. 1994, 3, 43-57.

32. LaScalza, S.; Arico, J.; Hughes, R. Effect of metal and sampling rate on accuracy of flock of birds electromagnetic tracking system. J. Biomech. 2003, 36, 141-144. [CrossRef] 
33. Milne, A.D.; Chess, D.G.; Johnson, J.A.; King, G.J. Accuracy of an electromagnetic tracking device: A study of the optimal range and metal interference. J. Biomech. 1996, 29, 791-793. [CrossRef]

34. Soltani, A.M.; Peric, M.; Francis, C.S.; Nguyen, T.T.; Chan, L.S.; Ghiassi, A.; Stevanovic, M.V.; Wong, A.K. The variation in the absence of the Palmaris longus in a multiethnic population of the united states: An epidemiological study. Plast. Surg. Int. 2012, 2012. [CrossRef] [PubMed]

35. Hardyck, C.; Petrinovich, L.F. Left-handedness. Psychol. Bull. 1977, 84, 385-404. [CrossRef] [PubMed]

36. Erić, M.; Koprivčić, I.; Vučinić, N.; Radić, R.; Krivokuća, D.; Lekšan, I.; Selthofer, R. Prevalence of the Palmaris longus in relation to the hand dominance. Surg. Radiol. Anat. 2011, 33, 481-484. [CrossRef] [PubMed]

37. Bergman, R.A.; Thompson, S.A.; Afifi, A.K. Catalog of Human Variation; Urban \& Schwarzenberg: Baltimore, MD, USA; München, Germany, 1984.

38. Erić, M.; Krivokuća, D.; Savović, S.; Leksan, I.; Vucinić, N. Prevalence of the Palmaris longus through clinical evaluation. Surg. Radiol. Anat. 2010, 32, 357-361. [CrossRef] [PubMed]

39. Venter, G.; van Schoor, A.N.; Bosman, M.C. Degenerative trends of the Palmaris longus muscle in a south african population. Clin. Anat. 2014, 27, 222-226. [CrossRef] [PubMed]

40. Azar, F.M.; Wright, P.E.; Canale, E. Flexor and extensor tendon injuries. In Campbell's Operative Orthopedics; Mosby: Maryland Heights, MI, USA, 2003; pp. 3423-3481.

41. Verdan, C.; Poulenas, I. Anatomic and functional relations between the tendons of the long palmar muscle and the long flexor muscle of the thumb at their crossing in the carpus. Ann. Chir. Plast. 1975, 20, 191-196. [PubMed]

42. Powell, B.W.; McLean, N.R.; Jeffs, J.V. The incidence of a palmaris longus tendon in patients with Dupuytren's disease. J. Hand Surg. Br. 1986, 11, 382-384. [CrossRef]

43. Holland, A.J.; McGrouther, D.A. Dupuytren's disease and the relationship between the transverse and longitudinal fibers of the palmar fascia: A dissection study. Clin. Anat. 1997, 10, 97-103. [CrossRef]

44. Chow, J.W.; Carlton, L.G.; Lim, Y.T.; Shim, J.H.; Chae, W.S.; Kuenster, A.F. Med. Sci. Sports Exerc. 1999, 31, 846-854. [PubMed]

45. Wei, S.H.; Chiang, J.Y.; Shiang, T.Y.; Chang, H.Y. Comparison of shock transmission and forearm electromyography between experienced and recreational tennis players during backhand strokes. Clin. J. Sport Med. 2006, 16, 129-135. [CrossRef] [PubMed]

46. Petersen, P.; Petrick, M.; Connor, H.; Conklin, D. Grip strength and hand dominance: Challenging the $10 \%$ rule. Am. J. Occup. Ther. 1989, 43, 444-447. [CrossRef] [PubMed]

47. Riemann, B.L.; Lephart, S.M. The sensorimotor system, part I: The physiologic basis of functional joint stability. J. Athl. Train. 2002, 37, 71-79. [PubMed]

48. Kelso, J.A.S. Planning and efferent components in the coding of movement. J. Mot. Behav. 1977, 9, $33-47$. [CrossRef] [PubMed]

(C) 2016 by the authors; licensee MDPI, Basel, Switzerland. This article is an open access article distributed under the terms and conditions of the Creative Commons Attribution (CC-BY) license (http://creativecommons.org/licenses/by/4.0/). 